\title{
Oxymatrine protects against sepsis-induced myocardial injury via inhibition of the TNF- $\alpha /$ p38-MAPK/caspase-3 signaling pathway
}

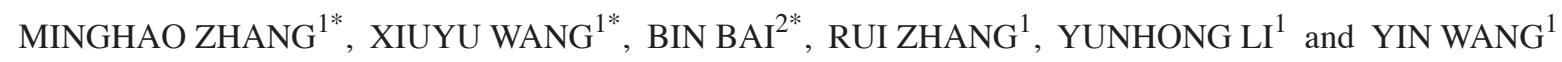 \\ ${ }^{1}$ Ningxia Key Laboratory of Cerebrocranial Diseases, Ningxia Medical University; ${ }^{2}$ Department of Endocrinology, \\ General Hospital of Ningxia Medical University, Yinchuan, Ningxia 750004, P.R. China
}

Received April 12, 2015; Accepted February 22, 2016

DOI: $10.3892 / \mathrm{mmr} .2016 .5250$

\begin{abstract}
Oxymatrine (OMT), which is a quinolizidine alkaloid extracted from the traditional Chinese herb Sophora flavescens Aiton, is often used to treat various inflammatory diseases. The present study aimed to investigate the protective effects of OMT against septic shock-induced myocardial injury in rats, and to determine the underlying mechanisms. In the present study, cecal ligation and puncture (CLP) was applied to generate a rat model of sepsis. The rats were randomly divided into six groups (n=8/group): Sham operation (CON) group, OMT control group, CLP model group, and CLP + OMT (high dose, $52 \mathrm{mg} / \mathrm{kg}$; medium dose, $26 \mathrm{mg} / \mathrm{kg}$; low dose, $13 \mathrm{mg} / \mathrm{kg}$ ) groups. Cardiac function and histological alterations were analyzed by light microscopy and electron microscopy. Myocardial cell apoptosis was detected by terminal deoxynucleotidyl transferase dUTP nick end labeling assay. The mRNA and protein expression levels were examined by reverse transcription-polymerase chain reaction and western blotting, respectively. Furthermore, the levels of tumor necrosis factor (TNF)- $\alpha$ in the myocardial tissue were determined by radioimmunoassay. The results demonstrated that OMT exhibited anti-inflammatory properties, improved myocardial contractility and compliance, and significantly decreased pathological injury to rat myocardial ultrastructure. In addition, OMT significantly decreased heart rate and left ventricular end diastolic pressure, and increased mean arterial pressure, left intraventricular pressure change rate, and left ventricular end systolic pressure in rats following septic shock. Treatment with OMT attenuated the mRNA expression of lipopolysaccharide binding protein, cluster of differentiation 14, nuclear factor (NF)- $\kappa \mathrm{B}(\mathrm{p} 65)$,
\end{abstract}

Correspondence to: Professor Yin Wang, Ningxia Key Laboratory of Cerebrocranial Diseases, Ningxia Medical University, 1160 Shengli Street, Yinchuan, Ningxia 750004, P.R. China

E-mail: yin-wang@hotmail.com

*Contributed equally

Key words: oxymatrine, septic shock, TNF- $\alpha /$ p38-MAPK/caspase-3 signaling pathway, myocardial injury
TNF- $\alpha$, p38-mitogen-activated protein kinase (MAPK) and caspase-3, and decreased the protein expression of $\mathrm{NF}-\kappa \mathrm{B}$ (p65), phosphorylated (p) NF- $\kappa$ B inhibitor- $\alpha$, p-p38-MAPK caspase- 3 and TNF- $\alpha$ in septic myocardial tissue. The present study concluded that OMT may offer substantial therapeutic potential for the treatment of septic shock-induced myocardial injury by inhibiting the TNF- $\alpha /$ p38-MAPK/caspase-3 signaling pathway.

\section{Introduction}

Sepsis and septic shock, which are caused by infection with gram-negative and gram-positive bacteria, fungi, viruses and parasites, have become increasingly important over the past few decades. Septic shock is a complex pathophysiological process, which is characterized by low blood pressure, systemic inflammatory reaction and multiple organ dysfunction. Patients suffering from sepsis utilize numerous medical resources, and the mortality rate is high and continuing to rise (1). The heart is considered a main 'target organ' of sepsis-induced multiple organ dysfunction, and the occurrence of myocardial dysfunction further promotes the development and deterioration of sepsis, which significantly contributes to the mortality of patients with septic shock (2). The pathogenesis of sepsis is very complex, and to date, studies regarding septic shock have concentrated on the generation of inflammatory mediators, including tumor necrosis factor (TNF)- $\alpha$ and interleukin-1 $\beta$, and damage to the liver, lungs and kidneys (3-6). In addition, previous studies have demonstrated that the heart is a potential site for the generation of inflammatory mediators $(7,8)$. Excessive production and release of proinflammatory mediators has an important role in the process of sepsis-induced myocardial dysfunction (SIMD) (9). Excessive production of TNF- $\alpha$, activation of the p38-mitogen-activated protein kinase (MAPK) pathway, and TNF- $\alpha$-mediated apoptosis have been reported to have an important role in the progression of SIMD; however, the mechanism by which numerous toxic products are released due to the generation and release of inflammatory mediators and biological active substances has yet to be elucidated. Our previous study detected marked inflammatory damage in the myocardial tissue of a rat model of sepsis (10). In addition, alterations to nuclear factor $(\mathrm{NF})-\kappa \mathrm{B}$ activity are closely associated with the 
production and release of inflammatory mediators in sepsis. The TNF- $\alpha /$ p38-MAPK/caspase-3 pathway has also been shown to be involved in the pathophysiological process of myocardial depression and myocardial cell apoptosis (11). These results suggested that inhibition of the expression of $\mathrm{NF}-\kappa \mathrm{B}$, the production of TNF- $\alpha$, and activation of the TNF- $\alpha /$ p38-MAPK/caspase-3 signaling pathway may have a positive role in the treatment of SIMD.

Oxymatrine (OMT) is an alkaloid extracted from Sophora flavescens. OMT exhibits various biological activities, and its anti-inflammatory effects have been reported in experimental animal models and clinical studies $(12,13)$. It has previously been demonstrated that OMT exerts inhibitory effects on Escherichia coli, Staphylococcus aureus, Salmonella typhimurium, Streptococcus agalactiae and Pasteurella multocida, and its effects are positively correlated with concentration (14). Furthermore, it has been reported that OMT may reduce the generation of oleic acid in a rat model of acute lung injury by inhibiting the p38-MAPK signaling pathway and the expression of TNF- $\alpha$ (15). In addition, by regulating the expression of lipopolysaccharide (LPS) recognition receptors [cluster of differentiation (CD)14 and scavenger receptor-A], OMT is able to reduce endotoxin-induced pathological lung damage in mice (16). However, it remains unclear whether OMT is able to prevent myocardial apoptosis associated with infectious shock by inhibiting activation of the TNF- $\alpha / \mathrm{p} 38-\mathrm{MAPK} /$ caspase- 3 signaling pathway. The present study used a cecal ligation and puncture (CLP) rat model to examine the effects of OMT on inhibition of the TNF- $\alpha / \mathrm{p} 38-\mathrm{MAPK} /$ caspase- 3 signaling pathway in response to septic shock-induced cardiac muscle injury.

\section{Materials and methods}

Chemicals and reagents. OMT was purchased from Ningxia Qi Yuan Pharmaceutical Co., Ltd. (Yinchuan, China). The terminal deoxynucleotidyl transferase dUTP nick end labeling (TUNEL) kit was obtained from Nanjing KeyGen Biotech Co., Ltd. (Nanjing, China). Immunoprecipitation cell lysis buffer and the bicinchoninic acid (BCA) protein concentration assay kit were purchased from Jiangsu Green Biotechnology Co., Ltd. (Xuzhou, China). The ${ }^{125}$ I TNF- $\alpha$ radioimmunoassay kit was obtained from Beijing Chemclin Biotech Co., Ltd. (Beijing, China). TRIzol ${ }^{\circledR}$ was purchased from Invitrogen; Thermo Fisher Scientific, Inc. (Waltham, MA, USA). Antibodies targeting lipopolysaccharide binding protein (LBP; goat polyclonal; cat. no. sc-70072), CD14 (goat polyclonal; cat. no. sc-5749), NF-кB (rabbit polyclonal; cat. no. sc-372), phosphorylated (p)-NF- $\mathrm{B}$ inhibitor (IкB)- $\alpha$ (goat polyclonal; cat. no. sc-7977), p-p38-MAPK (mouse monoclonal; cat. no. sc-7973) and caspase-3 (mouse monoclonal; cat. no. sc-7272)were purchased from Santa Cruz Biotechnology, Inc. (Dallas, TX, USA). Anti- $\beta$-actin (cat. no. A4700) was purchased from Sigma-Aldrich (St. Louis, MO, USA).

Animals. The animal experimental procedure used in the present study was approved by the Ningxia Medical University Animal Care Committee (Yinchuan, China). Male
Sprague-Dawley specific-pathogen-free rats [Animal Center, Ningxia Medical University, Yinchuan, China; SCXK (Ning) 2005-001], weighing 200-250 g, were randomly divided into six groups ( $\mathrm{n}=8 /$ group): Sham operation $(\mathrm{CON})$ group, OMT control group, CLP model group, and CLP + OMT (high dose, $52 \mathrm{mg} / \mathrm{kg}$; medium dose, $26 \mathrm{mg} / \mathrm{kg}$; low dose, $13 \mathrm{mg} / \mathrm{kg}$ ) groups. The septic shock model was induced by CLP, as previously described (17). The rats were maintained in light- and temperature-controlled conditions (12 h light/dark cycle; $22 \pm 2^{\circ} \mathrm{C}$ ) and were given ad libitum access to food and water. Briefly, rats were anesthetized with $40 \mathrm{mg} / \mathrm{kg}$ pentobarbital (2\%; Sigma-Aldrich) by intraperitoneal injection. A $2-3 \mathrm{~cm}$ midventral abdominal incision was made to expose the intestines. The ileocecal valve was ligated with 3-0 silk and three perforations were made in the cecum using a 20-gauge needle. To ensure consistent cecal damage among the animals, the perforated cecum was squeezed until 50-80 $\mu$ l feces extruded onto both surfaces, the bowel was reinserted into the abdomen and the incision was closed. In the sham group, the abdomen was opened to expose the intestines, and then closed. A single post-operative saline bolus was provided $(30 \mathrm{ml} / \mathrm{kg}$ subcutaneous) for fluid support. Caudal artery blood pressure was monitored; once it had decreased to $2 / 3$ basic blood pressure and $<20 \mathrm{mmHg}$, the model was considered successful. Rats received the drugs via tail vein injection (volume, $5 \mathrm{ml} / \mathrm{kg}$ ). In the sham and OMT control groups, rats received intravenous injection of normal saline and OMT $(26 \mathrm{ml} / \mathrm{kg})$, respectively, and the CLP group received normal saline $(26 \mathrm{ml} / \mathrm{kg})$. After treatment, the cecum was exposed under sterile conditions. The rats' tail artery pressure dropped to $2 / 3$ of the baseline blood pressure and pulse pressure was reduced to $<20 \mathrm{mmHg}$ as a judgment of sepsis model standards.

Cardiac function and histological analyses. To examine the cardiac function of mice in response to sepsis, the right common carotid artery was used to determine heart rate (HR), mean arterial pressure (MAP), left intraventricular pressure change rate $\left(\mathrm{LVdp} / \mathrm{dt}_{\max }\right)$, left ventricular end systolic pressure (LVESP) and left ventricular end diastolic pressure (LVEDP) using cardiac catheterization. Rats were anesthetized with sodium pentobarbital (40 mg/kg,i.p.), and were then sacrificed by cervical dislocation. Subsequently, the heart was removed for histological analyses. Apical tissue blocks $\left(\sim 2 \mathrm{~mm}^{3}\right)$ were collected, fixed in $10 \%$ formalin, and embedded in paraffin. After staining with hematoxylin and eosin, pathological alterations to the myocardial tissue were observed under a light microscope (CHC-212; Olympus Corporation, Tokyo, Japan). Other myocardial tissues $\left(\sim 2 \mathrm{~mm}^{3}\right)$ were placed in $2 \%$ glutaraldehyde, and were sectioned as electron microscopy specimens, in order to observe ultrastructural changes.

RT-PCR analysis. Cardiac tissues $(\sim 100 \mathrm{mg})$ were collected and homogenized using a Polytron PT1200E (Kinematica, Shanghai, China). Total RNA was extracted using TRIzol ${ }^{\circledR}$ reagent. The PCR reaction volume was $25 \mu 1$, and RT-PCR was conducted using the Promega One-Step RT-PCR kit (Promega Corporation, Madison, WI, USA). According to the protocol, $5 \mu \mathrm{l}$ DEPC water, $5 \mu \mathrm{l}$ AMV/TfI 5X buffer, $0.5 \mu \mathrm{l}$ dNTP mixture, $1 \mu 125 \mathrm{mM} \mathrm{MgSO}_{4}, 0.5 \mu \mathrm{l}$ Tf1 DNA polymerase, $0.5 \mu \mathrm{l}$ AMV-RT, $12.5 \mu \mathrm{l}$ Master Mix, $2.5 \mu 1$ 
Table I. Primer sequences, annealing temperature and product size of LBP, CD14, NF- $\kappa$ B (p65), TNF- $\alpha$, p38-MAPK, caspase-3 and $\beta$-actin genes.

\begin{tabular}{|c|c|c|c|}
\hline Gene & Sequences & Size (bp) & Annealing temperature $\left({ }^{\circ} \mathrm{C}\right)$ \\
\hline LBP & $\begin{array}{l}\text { F 5'ACTACAGTTTGGTGGCG3' } \\
\text { R 5'TTGTTGAAAGTTATTGAGGC3' }\end{array}$ & 500 & 55.3 \\
\hline CD14 & $\begin{array}{l}\text { F 5'ACATCTTGAACCTCCGCAAC3' } \\
\text { R 5'AGGGTTCCTATCCAGCCTGT3' }\end{array}$ & 500 & 59.2 \\
\hline $\mathrm{NF}-\kappa \mathrm{B}(\mathrm{p} 65)$ & $\begin{array}{l}\text { F 5'TGATGTGCATCAAGTGG3' } \\
\text { R 5'GAAGTTGAGTTTCGGGTAGGC3' }\end{array}$ & 296 & 58 \\
\hline TNF- $\alpha$ & $\begin{array}{l}\text { F 5'CAATGGCATGGATCTCAAAG3' } \\
\text { R 5'CAGAGCAATGACTCCAAAGT3' }\end{array}$ & 355 & 60 \\
\hline p38-MAPK & $\begin{array}{l}\text { F 5'CGTTGTTTCCTGGTACAGACC3' } \\
\text { R 5'CCATTTCTICTTGGTCAAGGG3 }\end{array}$ & 430 & 58 \\
\hline Caspase-3 & $\begin{array}{l}\text { F 5'ATGGACAACAACGAAACCTCCGTG3' } \\
\text { R 5'CCACTCCCAGTCATTCCTTTAGTG3' }\end{array}$ & 277 & 56 \\
\hline$\beta$-actin & $\begin{array}{l}\text { F 5'AGGTGAGAGGGAAATCGTGCG3' } \\
\text { R 5'CCACTCCCAGTCATTCCTTTAGTG3' }\end{array}$ & 662 & 55 \\
\hline
\end{tabular}

F, forward; R, reverse; LBP, lipopolysaccharide binding protein; CD14, cluster of differentiation 14; NF- $\mathrm{B}$, nuclear factor- $\mathrm{kB}$; TNF- $\alpha$, tumor necrosis factor- $\alpha$; $338-\mathrm{MAPK}, \mathrm{p} 38$-mitogen-activated protein kinase.

upstream primer (50 pmol), $2.5 \mu \mathrm{l}$ downstream primer (50 pmol) and RNA $(0.5-1 \mu \mathrm{g})$ were mixed in a microcentrifuge tube. RT-PCR was performed using GeneAmp 9700 thermal cycler (Applied Biosystems; Thermo Fisher Scientific, Inc., Waltham, MA, USA). First strand cDNA synthesis was conducted as follows: 1 cycle at $48^{\circ} \mathrm{C}$ for $45 \mathrm{~min}$ for RT, 1 cycle at $94^{\circ} \mathrm{C}$ for $2 \mathrm{~min}$ for AMV-RT inactivation and RNA/cDNA/primer denaturation. Second strand cDNA synthesis and PCR amplification were conducted as follows: 35 cycles at $94^{\circ} \mathrm{C}$ for $30 \mathrm{sec}$ for denaturation, annealing at the primer-specific temperatures for $1 \mathrm{~min}$, $68^{\circ} \mathrm{C}$ for $2 \mathrm{~min}$ for extension, and 1 cycle at $68^{\circ} \mathrm{C}$ for $7 \mathrm{~min}$ for final extension. $\beta$-actin was used as an internal control. The primers and annealing temperatures used are listed in Table I. Primers were designed using Primer 5.0 software (Premier Biosoft, Palo Alto, CA, USA), and were synthesized by Baisheng Company (Beijing, China). The PCR products were analyzed by $2 \%$ agarose gel electrophoresis using a Gel Doc $^{\mathrm{TM}}$ EZ system purchased from Bio-Rad Laboratories, Inc. (Hercules, CA, USA). The results were semi-quantified and expressed as relative optical density x surface area $\left(\mathrm{mm}^{2}\right)$. Relative mRNA expression levels were expressed as optical density normalized to $\beta$-actin.

Western blotting. Cardiac tissues $(\sim 100 \mathrm{mg})$ were collected and homogenized in radioimmunoprecipitation assay buffer (Biovision, Inc., Wuhan, China). Total protein concentration was measured using the BCA kit. Equal amounts of protein $(40 \mu \mathrm{g})$ were separated by $10 \%$ sodium dodecyl sulfate-polyacrylamide gel electrophoresis, and were then transferred to $0.45 \mu \mathrm{m}$ polyvinylidene difluoride membranes (KeHaoJia Company, Beijing, China). Blots were soaked in blocking buffer (5\% non-fat milk) and were then incubated with the various primary antibodies (LBP, 1:200; anti-CD14, 1:200; NF-кB, 1:500; p-IкB- $\alpha, 1: 200$; p-p38-MAPK, 1:300; caspase-3, $1: 300 ; \beta$-actin, $1: 400)$ at $4^{\circ} \mathrm{C}$ overnight. After thorough washing with Tris-buffered saline containing $0.1 \%$ Tween 20 , the membranes were incubated with horseradish peroxidase-conjugated anti-goat (cat. no. BA1060), anti-rabbit (cat. no. BA1054) and anti-mouse (cat. no. BA1051) secondary antibodies (1:10,000; Wuhan Boster Biological Technology Co., Ltd. (Wuhan, China), and immune complexes were visualized using an enhanced chemiluminescence detection system (Pierce Biotechnology, Inc., Rockford, IL, USA). The results were analyzed using a Gel Doc ${ }^{\mathrm{TM}}$ EZ system from Bio-Rad Laboratories, Inc. Expression levels were semi-quantified relative to the optical density of $\beta$-actin.

Electron microscopy. Apical sections of rat myocardial tissue $\left(\sim 2 \mathrm{~mm}^{3}\right)$ were collected and fixed in $2 \%$ glutaraldehyde for electron microscopy (H-600; Hitachi, Tokyo, Japan) in order to observe ultrastructural alterations to the myocardial tissue.

Radioimmunoassay. The levels of TNF- $\alpha$ in the myocardial tissue were determined by radioimmunoassay, according to the manufacturer's protocol. Myocardial tissue $(100 \mathrm{mg})$ was mixed with a threefold volume of phosphate-buffered saline and was homogenized. Subsequently, the homogenate was centrifuged at $35,616 \mathrm{x} \mathrm{g}$ for $20 \mathrm{~min}$ at $4^{\circ} \mathrm{C}$, and the protein levels of TNF- $\alpha$ in the supernatant were quantified using the radioimmunoassay assay kit.

TUNEL assay. The TUNEL assay was conducted according to the manufacturer's protocol. Briefly, myocardial tissue was fixed in $4 \%$ paraformaldehyde for $1 \mathrm{~h}$, and was processed for antigen retrieval with $0.1 \%$ Triton $\mathrm{X}-100$ and $0.1 \%$ sodium 
citrate for $2 \mathrm{~min}$ on ice. The tissue was subsequently incubated with a TUNEL reaction mixture containing terminal deoxynucleotidyl transferase in a humidified chamber for $1 \mathrm{~h}$ at $37^{\circ} \mathrm{C}$. Apoptosis of myocardial cells was characterized as reduced cell volume and visible brown particles within the nuclei. Myocardial cell apoptotic index (AI) was determined according to the following equation: $\mathrm{AI}=$ (total number of apoptotic cells/total number of cells) x $100 \%$.

Statistical analysis. Data are presented as the mean \pm standard error of the mean $(n=3)$. Data were analyzed by one-way analysis of variance followed by Student-Newman-Keuls post-hoc test for multiple comparisons. Statistical analyses were conducted using SPSS 11.5 (SPSS, Inc., Chicago, IL, USA). $\mathrm{P}<0.05$ was considered to indicate a statistically significant difference.

\section{Results}

Behavior and morphological alterations. Once the rats awoke from anesthesia, they started drinking and cleaning their fur. Rats in the CON and OMT groups exhibited a normal manner and appearance, as evidenced by a smooth and shiny coat and regular food intake. In addition, the rats in these groups did not appear dispirited or restless, and piloerection or chills were not observed. The abdominal organs also appeared normal. Rats in the CLP group gradually appeared listless, and exhibited reduced activity, food intake and responsiveness. In addition, the coat of the CLP rats appeared to have lost its luster. After 2-4 h, sickness gradually increased and the rats appeared slumped and restless, with increased piloerection, chills, diarrhea and eye secretions. After $9 \mathrm{~h}$, their condition worsened. Following an intraperitoneal cesarean section, there was visible liquid turbidity in the abdominal cavity; and the cecum emitted a foul-smelling odor, alongside swelling, necrosis, adhesion, and jejunal bowel distension. These findings are consistent with the literature (18), thus indicating that a rat model of sepsis was successfully generated. Rats in the CLP + OMT high and medium dose groups exhibited increased activity, and reduced dispiritedness, restlessness, piloerection and chills, as compared with the CLP group, following a laparotomy. In addition, there was no obvious liquid abnormality in the abdominal cavity, no cecal swelling, and no observation of gangrene and adhesion; however, improvement was not as obvious in the CLP + OMT low dose group.

Cardiac function assay. The CLP rat model was used to evaluate the effects of OMT on myocardial diastolic dysfunction, which is an important characteristic of septic shock in rats. As shown in Fig. 1, there was no difference in the cardiac function parameters, including HR, MAP, LVESP, LVEDP, $\mathrm{LVdp} / \mathrm{dt}_{\max }$ and $-\mathrm{LVdp} / \mathrm{dt}_{\max }$ indices, between the OMT control group and the CON group. In the CLP group, each index was significantly altered compared with the CON group. HR was increased by $18 \%$, MAP was reduced by $37 \%$, LVESP was reduced by $27 \%$, LVEDP was increased by $39 \%, \mathrm{LVdp} / \mathrm{dt}_{\max }$ was reduced by $38 \%$, and $-\mathrm{LVdp} / \mathrm{dt}_{\max }$ was reduced by $37 \%$. Following treatment with various doses of OMT, cardiac function improved. Compared with the CLP group, CLP + high dose OMT reduced HR by $10 \%(\mathrm{P}<0.05)$, increased MAP by 35\% ( $\mathrm{P}<0.01)$, increased LVESP by $28 \%(\mathrm{P}<0.01)$, reduced LVEDP by $25 \%(\mathrm{P}<0.05)$, increased $\mathrm{LVdp} / \mathrm{dt}_{\max }$ by $45 \%(\mathrm{P}<0.01)$, and increased $-\mathrm{LVdp} / \mathrm{dt}_{\max }$ by $29 \%(\mathrm{P}<0.01)$. CLP + medium dose OMT reduced HR by $8 \%(\mathrm{P}<0.05)$, increased MAP by $22 \%(\mathrm{P}<0.05)$, increased LVESP by $16 \%(\mathrm{P}<0.05)$, reduced LVEDP by $20 \%(\mathrm{P}<0.01)$, increased $\mathrm{LVdp} / \mathrm{dt}_{\max }$ by $32 \%(\mathrm{P}<0.01)$, and increased $-\mathrm{LVdp} / \mathrm{dt}_{\max }$ by $23 \%(\mathrm{P}<0.01) . \mathrm{CLP}+$ low dose OMT reduced HR by $4 \%(\mathrm{P}>0.05)$, increased MAP by $11 \%(\mathrm{P}<0.05)$, increased LVESP by $4 \%(\mathrm{P}>0.05)$, reduced LVEDP by $7 \%(\mathrm{P}>0.05)$, increased $\mathrm{LVdp} / \mathrm{dt}_{\max }$ by $13 \%(\mathrm{P}<0.05)$, and increased $-\mathrm{LVdp} / \mathrm{dt}_{\max }$ by $10 \%(\mathrm{P}<0.05)$.

Myocardial histological analysis. Myocardial histology appeared no different between the CON and OMT control groups. The endocardial membrane was complete, and edema and fibrous connective tissue hyperplasia were not detected. The myocardial stripes were clear and the nucleus was centered, with no vasodilation or inflammatory cell infiltration detected. Epicardial membrane integrity was intact, and was not covered with inflammatory exudate (Fig. 2A and B). The myocardial tissue of the CLP group exhibited an extensively disordered subendocardial structure compared with the CON group. A wide range of inflammatory cells infiltrated the tissue, and large numbers of mononuclear cells, intermingled with a few lymphocytes and neutrophils were detected. In addition, telangiectasia and bleeding were observed (Fig. 2C). The CLP group also displayed interstitial edema and fibroblast proliferation, and various degrees of cell necrosis and fibrosis. Myocardial tissue damage in the CLP + OMT low dose group was reduced to some degree; however, the myocardial structure remained slightly disorganized, with inflammatory cell infiltration, telangiectasia, bleeding, cell necrosis and fibrosis all detected (Fig. 2D). Myocardial tissue damage in the CLP + OMT medium and high dose groups was markedly reduced, and normal basic cardiac structure was observed. Edema, degeneration and necrosis were notably reduced; however, a small amount of inflammatory cell infiltrate and a few exudative changes remained (Fig. 2E and F). These results indicate that OMT may reduce myocardial injury and exert protective effects on cardiac structure and function in rats with septic shock.

Myocardial ultramicrohistological analysis. The myocardial tissue, including myofilaments, sarcomeres, capillaries, mitochondria, sarcoplasmic reticulum and nuclei, was not altered in the OMT control group compared with the CON group, and all structures appeared normal (Fig. 3A and B). The myofilament and sarcomere arrangement was normal, and the capillaries opened well. In addition, mitochondrial structures were normal, and mitochondria exhibited complete membranes, dense ridges and clear matrix. The structure of the intercalated disks was continuous. Sarcoplasmic reticulum was smooth and continuous. Nuclear structure was clear, with slightly visible nuclear pyknosis and uniform chromatin. Conversely, the ultramicrohistological structure of the CLP myocardial tissue was markedly degraded. As shown in Fig. 3C, mitochondria appeared markedly swollen, resulting in damaged membranes and disordered cristae. Disordered 


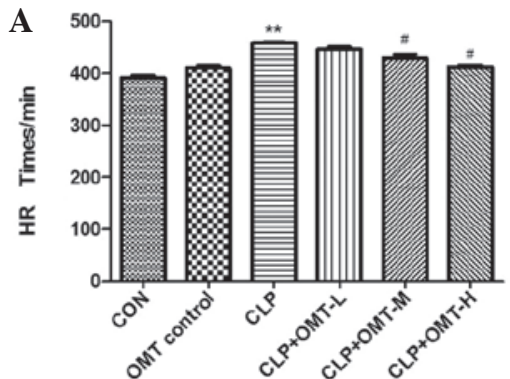

D

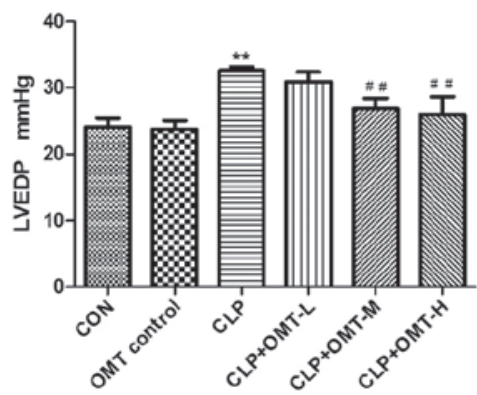

B

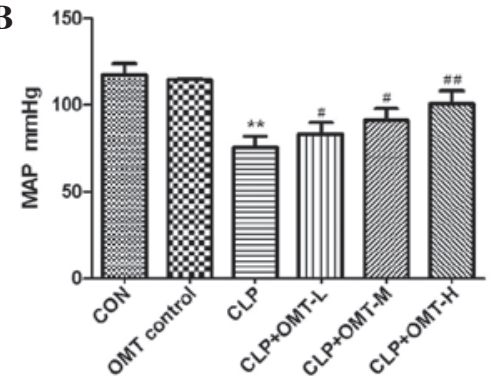

$\mathbf{E}$

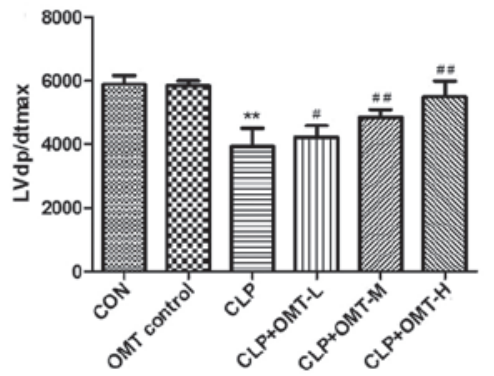

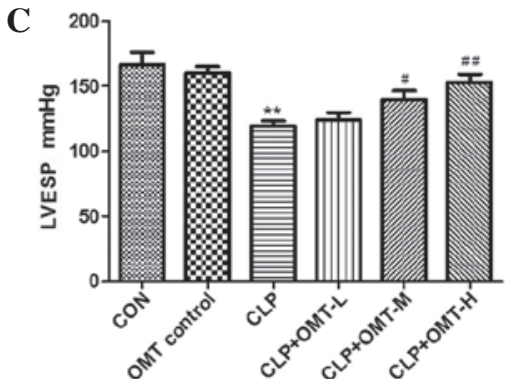

F

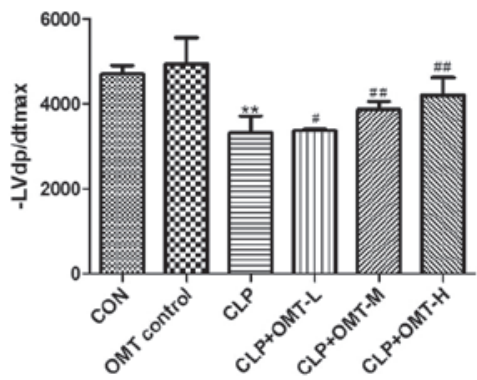

Figure 1. Effects of oxymatrine (OMT) on cardiac function in rats with septic shock (n=8). The following indices were measured: (A) Heart rate (HR), (B) mean arterial pressure (MAP), (C) left ventricular end systolic pressure (LVESP), (D) left ventricular end diastolic pressure (LVEDP), (E) left intraventricular pressure change rate $\left(\mathrm{LVdp} / \mathrm{dt}_{\max }\right)$ and $(\mathrm{F})-\mathrm{LVdp} / \mathrm{dt}_{\max }$ by cardiac catheterization in all groups, including the control (CON), OMT control, cecal ligation and puncture (CLP), CLP + OMT low dose (L), CLP + OMT medium dose (M), CLP + OMT high dose (H) groups. Data are presented as the mean \pm standard error of the mean. ${ }^{* *} \mathrm{P}<0.01$ compared with the CON group; ${ }^{\#} \mathrm{P}<0.05$ and ${ }^{\# \#} \mathrm{P}<0.01$ compared with the CLP group.

myofilaments and sarcomeres subsequently dissolved, leading to the generation of vacuoles. The nuclei shrunk in size and the chromatin was marginated. The structure of the intercalated disks was not continuous, and dissolution was observed. However, the ultramicrohistological injuries of the myocardial tissue were markedly improved in the CLP + OMT groups (Fig. 3D-F). The myocardial fibers were normally arranged, and the majority of mitochondria exhibited a complete structure with ordered dense ridges. Although the structure of the mitochondria cristae appeared vague, arrangement remained regular. In addition, mitochondrial swelling was reduced; however, in some cases mitochondrial damage was not yet fully recovered.

Effects of OMT on LBP, CD14, NF- $\kappa B$ (p65), TNF- $\alpha$, p38-MAPK and caspase-3 mRNA expression. The mRNA expression levels of LBP, CD14, NF- $\kappa$ B (p65), TNF- $\alpha$, p38-MAPK and caspase-3 were similar in the myocardial tissue of the OMT control and CON groups. However, expression levels were significantly increased in the CLP group $(\mathrm{P}<0.05)$. Compared with the CLP group, the mRNA expression levels of LBP, CD14, NF- $\mathrm{BB}$ (p65), TNF- $\alpha$, p38-MAPK and caspase- 3 were markedly decreased in the CLP + OMT groups ( $\mathrm{P}<0.05$; Fig. 4).

Effects of OMT on NF- $\kappa B$ (p65), p-I $\kappa B-\alpha, p-p 38-M A P K$ and caspase-3 protein expression. Western blotting results indicated that NF- $\kappa \mathrm{B}$ (p65), p-IкB- $\alpha, \mathrm{p}-\mathrm{p} 38-\mathrm{MAPK}$ and caspase-3 protein expression levels were similar in the myocardial tissue of the OMT control and CON groups. However, expression levels were significantly increased in the CLP group $(\mathrm{P}<0.05)$. Conversely, NF- $\mathrm{B}$ (p65), p-IкB- $\alpha, \mathrm{p}-\mathrm{p} 38-\mathrm{MAPK}$ and caspase-3 protein expression levels were markedly decreased in the CLP + OMT groups compared with in the CLP group $(\mathrm{P}<0.05$; Fig. 5).

Effects of OMT on TNF- $\alpha$ expression. The results of a radioimmunoassay demonstrated that $\mathrm{TNF}-\alpha$ levels were significantly increased in the CLP group compared with in the CON and OMT control groups $(\mathrm{P}<0.05)$. However, compared with the CLP group, the TNF- $\alpha$ levels were significantly decreased in the CLP + OMT groups $(\mathrm{P}<0.05)$ (Fig. 6A).

Effects of OMT on myocardial cell apoptosis. No significant differences were detected in myocardial cell AI between the $\mathrm{CON}$ and OMT control groups. Compared with the CON group, myocardial AI was significantly increased in the CLP group $(\mathrm{P}<0.01)$. Conversely, myocardial AI was significantly reduced following treatment with various doses of OMT compared with in the CLP group $(\mathrm{P}<0.05)$ (Fig. 6B).

\section{Discussion}

OMT, which is a type of alkaloid extracted from Sophora flavescens Ait., has previously been reported to exert positive pharmacological effects on regulation of the immune response, reduction of hypersensitive reactions, and inhibition of histamine release in vitro and in vivo (19-21). Furthermore, OMT has been demonstrated to inhibit LPS-induced inflammation by downregulating Toll-like receptor (TLR)4/NF- $\kappa \mathrm{B}$ in macrophages (22). OMT also exerts protective effects on LPS-induced mastitis, an inflammatory reaction of the mammary gland, the anti-inflammatory mechanism of which has been shown to be associated with inhibition of the NF- $\mathrm{NB}$ and MAPK signaling pathways (23). However, the pharmacological effects of OMT 

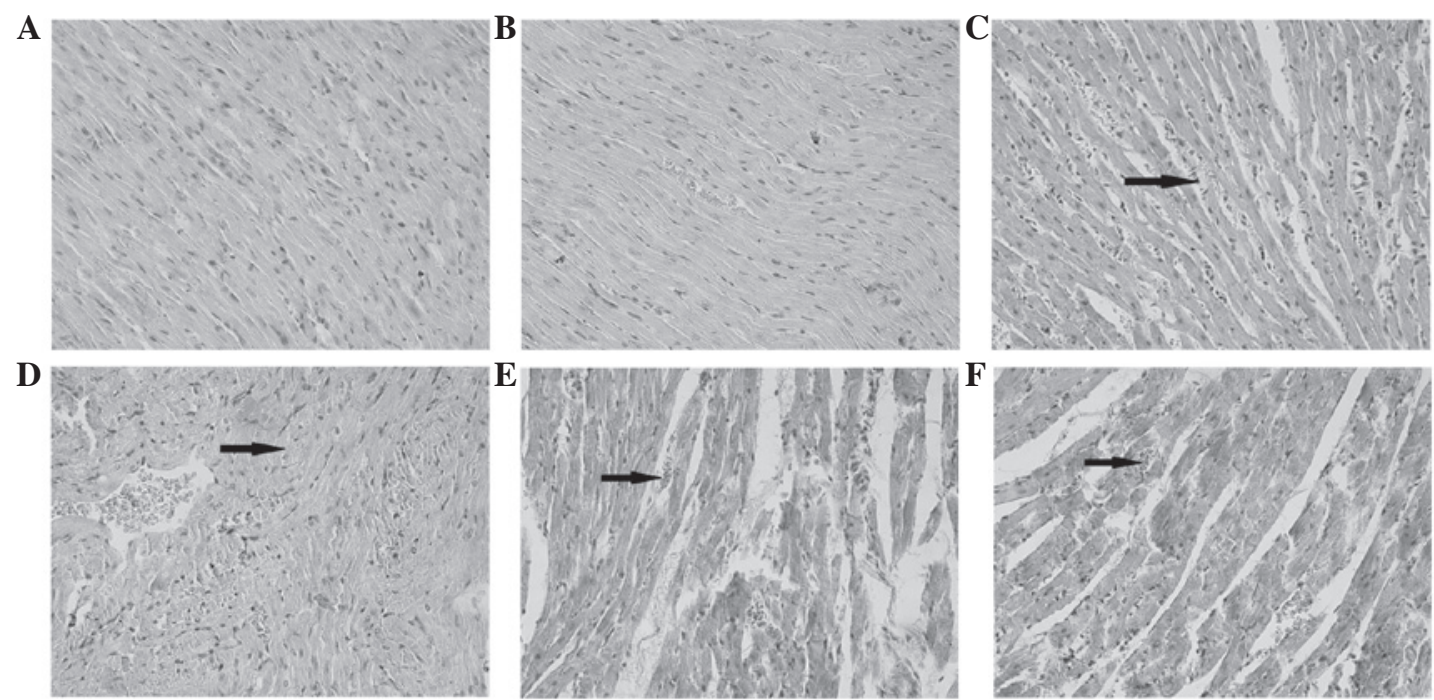

Figure 2. Myocardial histological analyses of rats with septic shock [hematoxylin and eosin (HE); magnification, x200]. The apical heart tissue blocks $\left(\sim 2 \mathrm{~mm}{ }^{3}\right)$ were collected and fixed in $10 \%$ formalin, then embedded in paraffin, and stained with HE for histological analyses. (A) Sham operation control group received intravenous injection of normal saline. (B) Oxymatrine (OMT) control group received intravenous injection of $26 \mathrm{ml} / \mathrm{kg}$ OMT. (C) Cecal ligation and puncture (CLP) group received intravenous injection of normal saline $(26 \mathrm{ml} / \mathrm{kg})$ and underwent CLP. (D) CLP + OMT low dose group, CLP rats received a tail vein injection of $13 \mathrm{mg} / \mathrm{kg}$ OMT. (E) CLP + OMT medium dose group, CLP rats received a tail vein injection of $26 \mathrm{mg} / \mathrm{kg}$ OMT. (F) CLP + OMT high dose group, CLP rats received a tail vein injection of $52 \mathrm{mg} / \mathrm{kg}$ OMT. Arrows indicate a gradual reduction in myocardial histological changes.

A
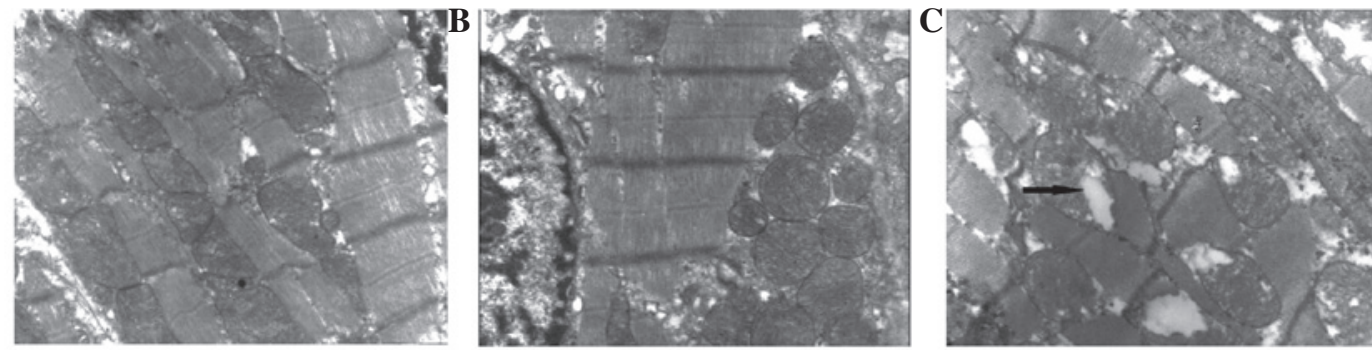

D
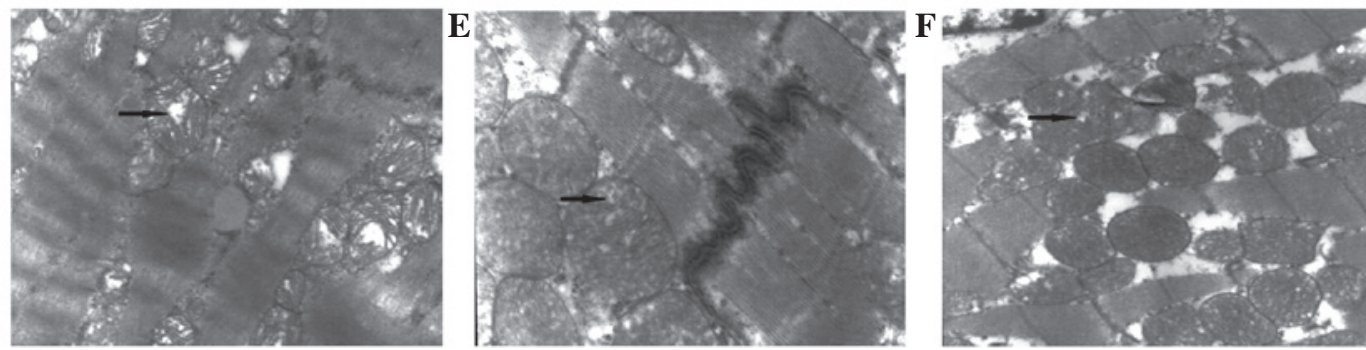

Figure 3. Myocardial ultramicrohistological analyses (magnification, x12,000). Myocardial tissues $\left(\sim 2 \mathrm{~mm}^{3}\right)$ were placed in $2 \%$ glutaraldehyde, and were sectioned as electron microscopy specimens, in order to observe ultrastructural changes. (A) Control group; (B) oxymatrine (OMT) control group; (C) cecal ligation and puncture (CLP) group; (D) CLP + OMT low dose group; (E) CLP + OMT medium dose group; (F) CLP + OMT high dose group. Arrows indicate a gradual reduction in myocardial damage.

on cardiac tissue and the underlying mechanisms have not been well elucidated. Zhang et al (24) demonstrated that OMT was able to ameliorate left ventricular hypertrophy and dysfunction in rats with heart failure. In addition, a combination of sodium ferulate and OMT has been reported to exert protective effects against CLP-induced lethal sepsis in mice (25). Accordingly, the present study used the CLP-induced model of sepsis to investigate the anti-inflammatory effects of OMT, and aimed to determine its protective effects and possible underlying mechanisms on sepsis-induced cardiac injury.

Sepsis is a complex pathophysiological process that is characterized by hypotension, hypoxia, metabolic acidosis, systemic inflammatory response and multiple organ dysfunction. The heart is considered a potential site for the generation of inflammatory mediators, and the generation and release of proinflammatory mediators, such as TNF- $\alpha$, and activation of the p38-MAPK pathway has been reported to have an important role in sepsis-induced cell apoptosis and cardiac dysfunction (26). A previous study confirmed that the three pathways: Janus kinase/signal transducers and activators of transcription, $\mathrm{NF}-\kappa \mathrm{B}$ and MAPK are important in the regulation of inflammatory signal transduction (27). NF- $\kappa \mathrm{B}$ is an important cell transcription factor associated with immune and inflammatory reactions, which has a core position in the convergence of these 
A
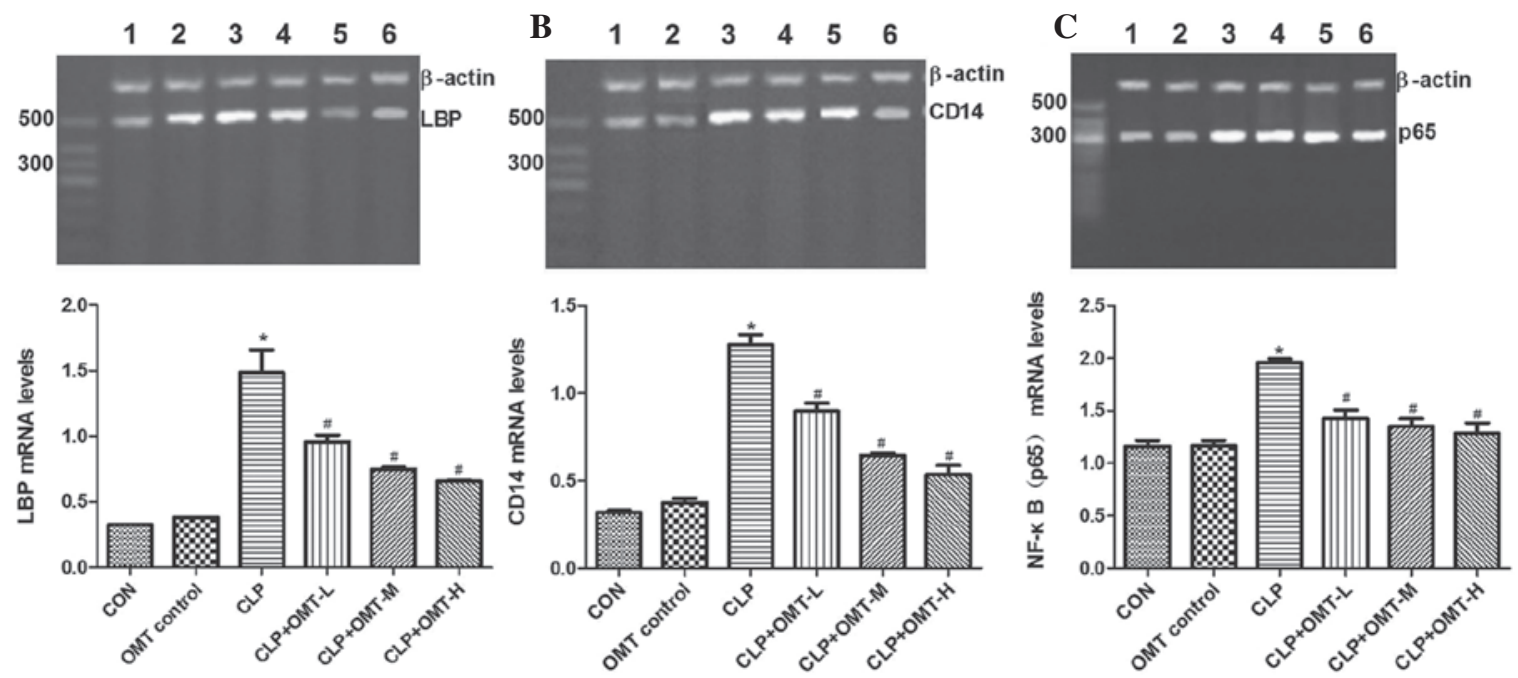

D

$\mathbf{E}$

$\mathbf{F}$
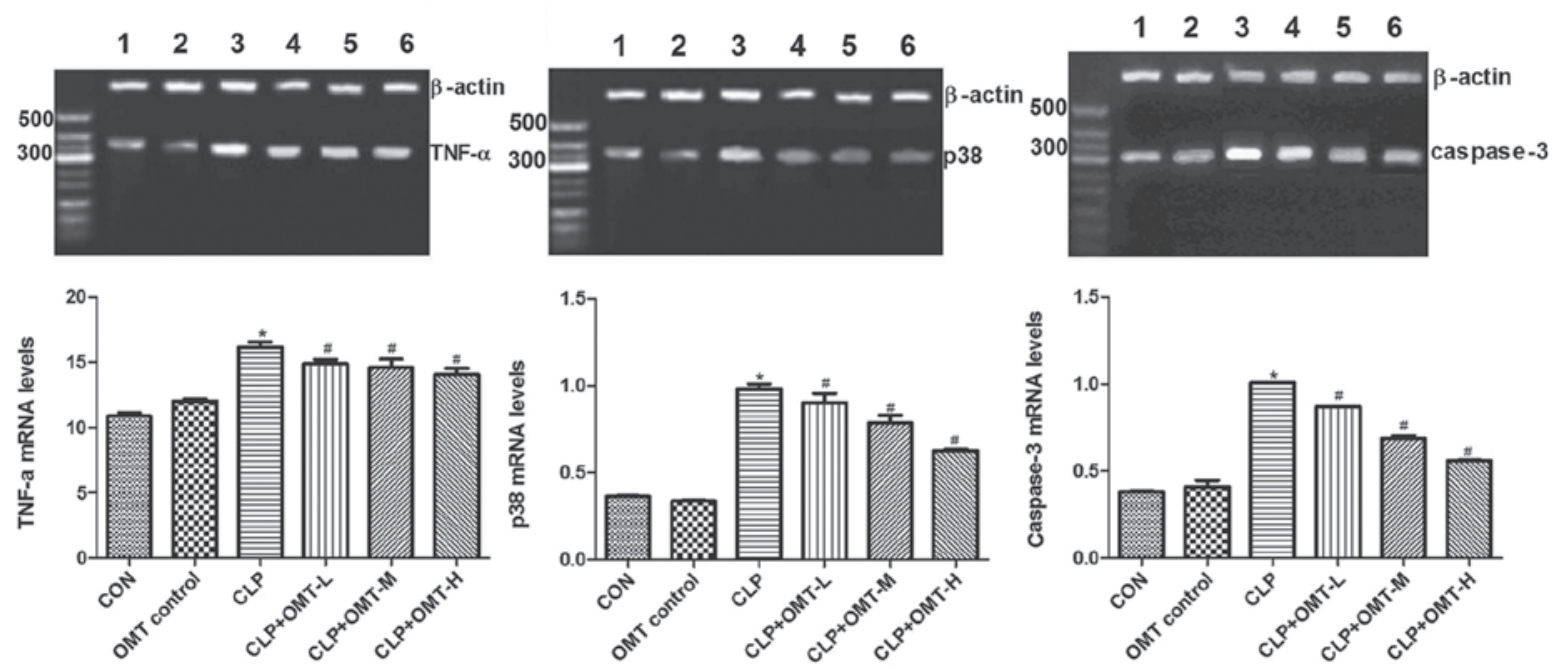

Figure 4. Effects of oxymatrine (OMT) on (A) lipopolysaccharide binding protein (LBP), (B) cluster of differentiation (CD)14, (C) nuclear factor- $\mathrm{kB}$ (p65), (D) tumor necrosis factor (TNF)- $\alpha$, (E) p38-mitogen-activated protein kinase and (F) caspase-3 mRNA expression in rat cardiac muscle with septic shock. Lane 1, Control (CON) group; lane 2, OMT control group; lane 3, cecal ligation and puncture (CLP) group; lane 4, CLP + OMT low dose (L) group; lane 5, CLP + OMT medium dose (M) group; lane 6, CLP + OMT high dose (H) group. Polymerase chain reaction results were quantified, and bar graphs present the mRNA expression levels relative to the internal control ( $\beta$-actin). Data are presented as the mean \pm standard error of the mean. ${ }^{*} \mathrm{P}<0.05$ compared with the CON group; ${ }^{*} \mathrm{P}<0.05$ compared with the CLP group.

three intracellular signaling pathways (28). In the resting state, $\mathrm{NF}-\kappa \mathrm{B}$ proteins combine with the I $\mathrm{B}$ family proteins, and thus exist in the cytoplasm in their inactive form. Inactive $N F-\kappa B$ complex and active $\mathrm{NF}-\kappa \mathrm{B}$ are in dynamic equilibrium between the cytoplasm and nucleus.

When gram-negative bacteria infect the body and die they release LPS. LPS is the main pathogenic gram-negative bacteria-associated molecular pattern, which is recognized by a series of receptors in the innate immune system, including LBP, CD14 and TLR4, resulting in activation of effector cells. LPS is known to first combine with LBP and CD14 in the circulating blood, thus forming the LPS-LBP-CD14 complex. This complex is then transported to the cell membrane, where it is anchored to the LPS receptor complex, and induces dimerization of TLR4 and two molecules of the extracellular adapter protein MD-2. The cytoplasmic region of TLR4 then interacts with the adaptor protein myeloid differentiation factor 88 , inducing the activation of NF- $\kappa \mathrm{B}$-inducing kinase (NIK) (29). NIK is a serine/threonine protein kinase, which can phosphorylate I $\kappa \mathrm{B}$ kinase and induce its activation, consequently leading to I $\kappa$ B- $\alpha$ phosphorylation. Phosphorylation of I $\mathrm{B}$ - $\alpha$ induces $\mathrm{I} \kappa \mathrm{B}$ and $\mathrm{NF}-\kappa \mathrm{B}$ dissociation, thus resulting in nuclear localization of $\mathrm{NF}-\kappa \mathrm{B}$ through the nuclear pore where it binds to corresponding DNA-specific sequences, so as to play its role in the regulation of gene transcription. $\mathrm{NF}_{-} \kappa \mathrm{B}$ can bind to the fixed nucleotide sequence of subregions of numerous cytokines, and the promoters of inflammatory mediators, thus initiating gene expression of cytokines such as TNF- $\alpha$ (30). Upregulation of TNF- $\alpha$ eventually activates caspase- 3 and induces myocyte apoptosis via the $\mathrm{NF}-\kappa \mathrm{B}$ signaling pathway.

The present study detected the mRNA expression levels of LBP, CD14, NF- $\kappa$ B (p65), TNF- $\alpha$, p38-MAPK and caspase-3, and the protein expression levels of $\mathrm{NF}-\kappa \mathrm{B}(\mathrm{p} 65), \mathrm{p}-\mathrm{I} \kappa \mathrm{B}-\alpha$, p-p38 MAPK, caspase-3 and TNF- $\alpha$ in the myocardial tissue of rats with CLP-induced sepsis. The expression of all of these factors was significantly increased in the CLP group. In addition, HR and LVEDP were markedly increased, whereas 
A

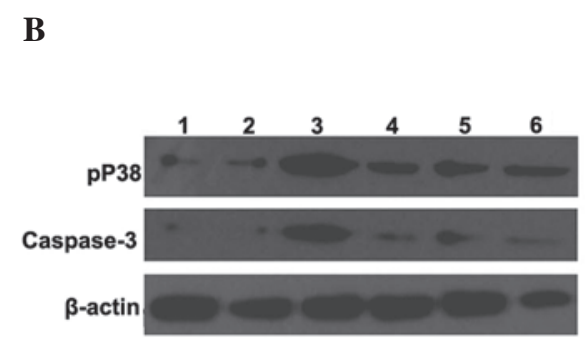

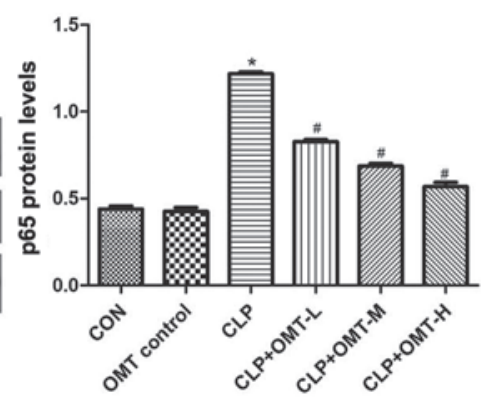

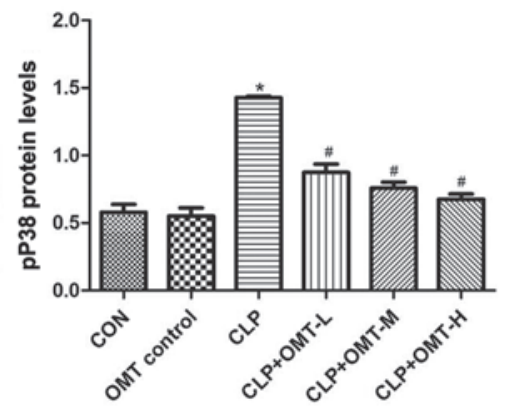

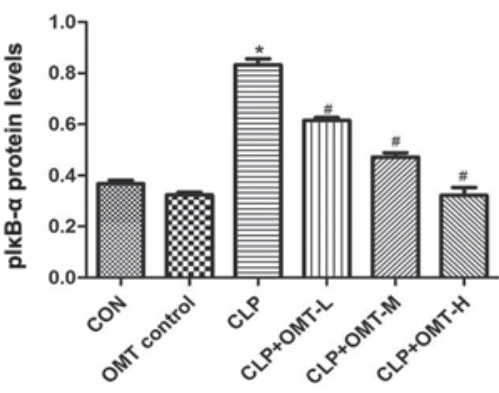

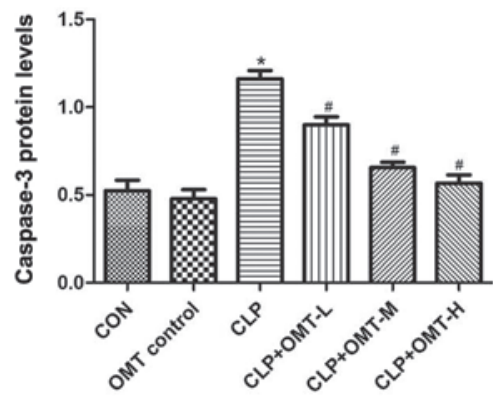

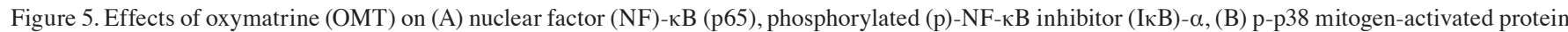
kinase and caspase-3 protein expression. Western blotting was used to determine protein expression, with $\beta$-actin used as an internal control. Lane 1 , Control (CON) group; lane 2, OMT control group; lane 3, cecal ligation and puncture (CLP) group; lane 4, CLP + OMT low dose (L) group; lane 5, CLP + OMT medium dose (M) group; lane 6, CLP + OMT high dose (H) group. Western blotting was semi-quantified and the bar graphs present the relative protein expression levels. Data are presented as the mean \pm standard error of the mean. ${ }^{*} \mathrm{P}<0.05$ compared with the CON group; ${ }^{*} \mathrm{P}<0.05$ compared with the CLP group.

A

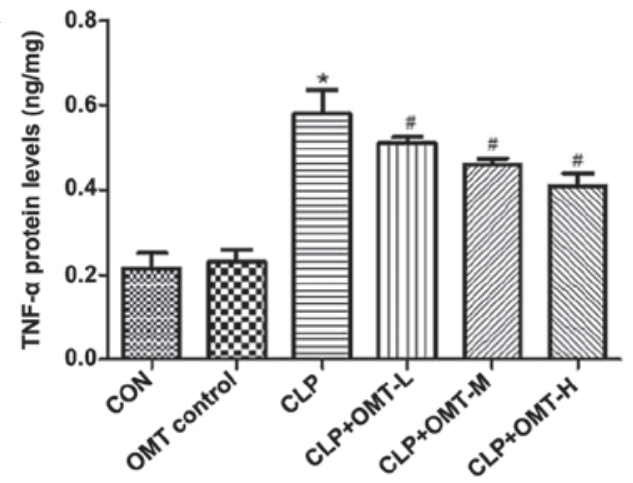

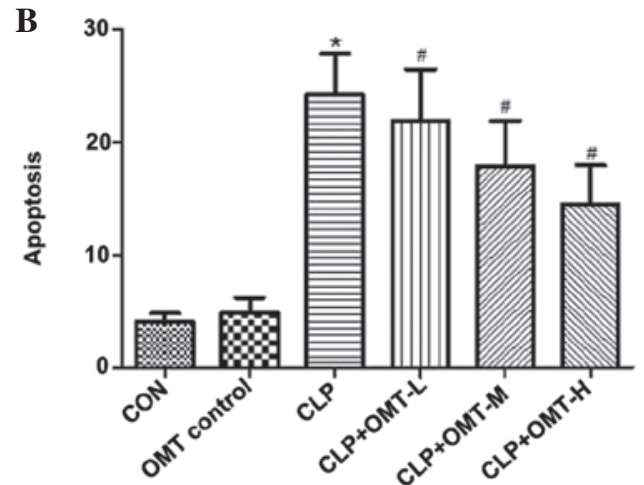

Figure 6. Effects of oxymatrine (OMT) on tumor necrosis factor (TNF)- $\alpha$ protein expression and apoptosis in cardiac muscle from rats with septic shock. (A) Results of a TNF- $\alpha$ radioimmunoassay. (B) Results of a terminal deoxynucleotidyl transferase dUTP nick end labeling assay of myocardial cell apoptosis. Apoptosis of myocardial cells was characterized as reduced cell volume and visible brown particles within the nuclei. Myocardial cell apoptotic index (AI) was determined as follows AI = (total number of apoptotic cells/total number of cell) $\mathrm{x} 100 \%$. Data are presented as the mean \pm standard error of the mean. ${ }^{*} \mathrm{P}<0.05$ compared with the CON group; ${ }^{*} \mathrm{P}<0.05$ compared with the CLP group.

MAP, LVESP, LVdp/dt $t_{\max }$ and $-L V d p / \mathrm{dt}_{\max }$ were decreased. These results suggested that cardiac structure and function were markedly damaged, and the underlying mechanism may be associated with activation of LBP and CD14 by toxins, such as LPS, on the myocardial cell membrane.

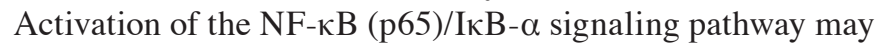
result in increased production of TNF- $\alpha$ and other inflammatory mediators, and activation of the p38-MAPK/caspase-3 pathway may induce myocardial cell apoptosis.

Following intervention with various doses of OMT, the mRNA expression levels of LBP, CD14, NF- $\kappa$ B (p65) and TNF- $\alpha$ were significantly decreased in myocardial tissue compared with in the CLP group, thus indicating that OMT exerts marked inhibitory effects on the LBP, CD14 and NF- $\mathrm{kB}$ pathway in the myocardial tissue of rats with sepsis. The underlying mechanism of these effects may be associated with suppression of NF- $\mathrm{kB}$ (p65) expression and NIK activation, thereby inhibiting the expression of proinflammatory cytokines such as TNF- $\alpha$. In addition, the mRNA expression levels of p38-MAPK and caspase-3 mRNA were significantly decreased, which was consistent with the decreased protein expression levels of p-p38-MAPK and caspase-3 detected in the myocardial tissue. Furthermore, following OMT treatment, cardiac function, myocardial tissue structure and ultrastructural injury were all markedly improved, and the myocardial AI was significantly reduced. These results indicated that OMT may increase myocardial contractility and compliance, correct increases in LVEDP caused by apoptosis of myocardial cells, and decrease preload, thus improving myocardial function. The underlying mechanisms of these effects may involve inhibition of TNF- $\alpha$ 
expression and reduced TNF- $\alpha$-induced myocardial apoptosis, which is mediated by the p38-MAPK/caspase-3 pathway.

The present study is the first, to the best of our knowledge, to demonstrate that sepsis-induced cardiac injuries are associated with the TNF- $\alpha / \mathrm{p} 38$-MAPK/caspase-3 signaling pathway, and that OMT has the ability to suppress activation of the p38-MAPK/caspase-3 pathway. Therefore, OMT may be considered a potential therapeutic agent for the treatment of septic shock.

In conclusion, the present study provides valuable information regarding the mechanisms underlying the cardioprotective effects of OMT, thus providing a theoretical basis for further clinical studies focusing on the treatment of sepsis.

\section{Acknowledgements}

The present study was supported by grants from the Natural Science Foundation of Ningxia (grant nos. NZ13068 and NZ14057), the National Natural Science Foundation of China (grant nos. 31260243 and 31460257), and the Ningxia Higher School Scientific Research Project (grant no. NGY2013081). Additional funding was provided to Dr Yin Wang by the Program for New Century Excellent Talents in University.

\section{References}

1. Dellinger RP, Levy MM, Rhodes A, Annane D, Gerlach H, Opal SM, Sevransky JE, Sprung CL, Douglas IS, Jaeschke R, et al; Surviving Sepsis Campaign Guidelines Committee including The Pediatric Subgroup: Surviving Sepsis Campaign: International guidelines for management of severe sepsis and septic shock, 2012. Intensive Care Med 39: 165-228, 2013

2. Nowak RM, Nanayakkara P, DiSomma S, Levy P, Schrijver E, Huyghe R, Autunno A, Sherwin RL, Divine G and Moyer M: Noninvasive hemodynamic monitoring in emergency patients with suspected heart failure, sepsis and stroke: The PREMIUM registry. West J Emerg Med 15: 786-794, 2014.

3. Celes MR, Prado CM and Rossi MA: Sepsis: Going to the heart of the matter. Pathobiology 80: 70-86, 2012.

4. Zhang MH, Li GZ, Xu H, Zhang J and Cao J: Effect of oxymatrine on NF-kappaB and other cell factors in rats lung tissue with septic shock. Zhongguo Zhong Yao Za Zhi 33: 2390-2394, 2008 (In Chinese).

5. Zhang MH, Xu H, Wang F, Yang XL, Zhang J, Li GZ and Cao J: The preventive and therapeutic effects of oxymatrine on lung injury in a rat model of septic shock. Ningxia Yixueyuan Xuebao 30: 421-423, 2008 (In Chinese)

6. Wang XY, Zhang MH, Yang ML, Jiang YD, Li GZ, Yang XL, $\mathrm{Xu} \mathrm{H}$ and Cao J: Effect of oxymatrine on JAK2/STAT3 signaling in renal tissues of rats with septic shock. Zhongguo Zhong Yao Za Zhi 38: 2696-2700, 2013 (In Chinese).

7. Belperio J, Horwich T, Abraham WT, Fonarow GC, Gorcsan J III, Bersohn MM, Singh JP, Sonel A, Lee LY, Halilovic J, et al: Inflammatory mediators and clinical outcome in patients with advanced heart failure receiving cardiac resynchronization therapy. Am J Cardiol 117: 617-625, 2016.

8. Pecoraro M, Del Pizzo M, Marzocco S, Sorrentino R, Ciccarelli M, Iaccarino G, Pinto A and Popolo A: Inflammatory mediators in a short-time mouse model of doxorubicin-induced cardiotoxicity. Toxicol Appl Pharmacol 293: 44-52, 2016.

9. Moussa MD, Santonocito C, Fagnoul D, Donadello K, Pradier O, Gaussem P, De Backer D and Vincent JL: Evaluation of endothelial damage in sepsis-related ARDS using circulating endothelial cells. Intensive Care Med 41: 231-238, 2015.

10. Zhang $M$, Wang $X$, Wang $X$, Hou X, Teng P, Jiang Y, Zhang L, Yang $\mathrm{X}$, Tian J, Li G, et al: Oxymatrine protects against myocardial injury via inhibition of JAK2/STAT3 signaling in rat septic shock. Mol Med Rep 7: 1293-1299, 2013.
11. Drosatos K, Lymperopoulos A, Kennel PJ, Pollak N, Schulze PC and Goldberg IJ: Pathophysiology of sepsis-related cardiac dysfunction: Driven by inflammation, energy mismanagement, or both? Curr Heart Fail Rep 12: 130-140, 2015.

12. Yuan X, Wang Y, Du D, Hu Z, Xu M, Xu M and Liu Z: The effects of the combination of sodium ferulate and oxymatrine on lipopolysaccharide-induced acute lung injury in mice. Inflammation 35: 1161-1168, 2012.

13. Cui HL, Wang YF, Li XL and Kang QX: Clinical observation of matrine injection in the treatment of 51 cases of various types of cancers. Shanxi Yiyao Zazhi 22: 232-233, 1993 (In Chinese)

14. Zheng P, Niu FL, Liu WZ, Shi Y and Lu LG: Anti-inflammatory mechanism of oxymatrine in dextran sulfate sodium-induced colitis of rats. World J Gastroenterol 11: 4912-4915, 2005.

15. Xu GL, Yao L, Rao SY, Gong ZN, Zhang SQ and Yu SQ: Attenuation of acute lung injury in mice by oxymatrine is associated with inhibition of phosphorylated p38 mitogen-activated protein kinase. J Ethnopharmacol 98: 177-183, 2005.

16. Han Y, Zhou Y and Liu Q: Antiendotoxic effects of Sophora alopecuroides L. Zhong Yao Cai 29: 1066-1068, 2006 (In Chinese).

17. Jing HM: Cecal ligation puncture of rat model of sepsis. Zhongguo Chaosheng Yixue Zazhi 2: 126-127, 1990 (In Chinese).

18. Zhang L, Yao J, Wang X, Li H, Liu T and Zhao W: Poly (ADP-ribose) synthetase inhibitor has a heart protective effect in a rat model of experimental sepsis. Int J Clin Exp Pathol 8: 9824-9835, 2015

19. Fan DL, Zhao WJ, Wang YX, Han SY and Guo S: Oxymatrine inhibits collagen synthesis in keloid fibroblasts via inhibition of transforming growth factor- $\beta 1 / \mathrm{Smad}$ signaling pathway. Int J Dermatol 51: 463-472, 2012.

20. Liu L, Lu W, Ma Z and Li Z: Oxymatrine attenuates bleomycin-induced pulmonary fibrosis in mice via the inhibition of inducible nitric oxide synthase expression and the TGF- $\beta / \mathrm{Smad}$ signaling pathway. Int J Mol Med 29: 815-822, 2012.

21. Chai NL, Fu Q, Shi H, Cai CH, Wan J, Xu SP and Wu BY: Oxymatrine liposome attenuates hepatic fibrosis via targeting hepatic stellate cells. World J Gastroenterol 18: 4199-4206, 2012.

22. Zhang Y, Yan $\mathrm{R}$ and $\mathrm{Hu}$ Y: Oxymatrine inhibits lipopolysaccharide-induced inflammation by down-regulating Toll-like receptor 4/nuclear factor-kappa B in macrophages. Can J Physiol Pharmacol 93: 253-260, 2015.

23. Yang Z, Yin R, Cong Y, Yang Z, Zhou E, Wei Z, Liu Z, Cao Y and Zhang N: Oxymatrine lightened the inflammatory response of LPS-induced mastitis in mice through affecting NF- $\kappa \mathrm{B}$ and MAPKs signaling pathways. Inflammation 37: 2047-2055, 2014.

24. Zhang W, Zhang J, Liu YK, Liu J, Wang X, Xu Q, Wang Y, $\mathrm{Xu} \mathrm{X}$ and Dai G: Cardioprotective effects of oxymatrine on isoproterenol-induced heart failure via regulation of DDAH/ADMA metabolism pathway in rats. Eur J Pharmacol 745: 29-35, 2014.

25. Xu M, Wang W, Pei X, Sun S, Xu M and Liu Z: Protective effects of the combination of sodium ferulate and oxymatrine on cecal ligation and puncture-induced sepsis in mice. Exp Ther Med 7: 1297-1304, 2014

26. Ailawadi S, Wang X, Gu H and Fan GC: Pathologic function and therapeutic potential of exosomes in cardiovascular disease. Biochim Biophys Acta 1852: 1-11, 2015.

27. Park SY, Bae YS, Ko MJ, Lee SJ and Choi YW: Comparison of anti-inflammatory potential of four different dibenzocyclooctadiene lignans in microglia; action via activation of PKA and Nrf-2 signaling and inhibition of MAPK/STAT/NF- $\kappa \mathrm{B}$ pathways. Mol Nutr Food Res 58: 738-748, 2014.

28. Kim BH,Lee JM, Jung YG, Kim S and Kim TY: Phytosphingosine derivatives ameliorate skin inflammation by inhibiting NF- $\kappa \mathrm{B}$ and JAK/STAT signaling in keratinocytes and mice. J Invest Dermatol 134: 1023-1032, 2014.

29. Park OJ, Han JY, Baik JE, Jeon JH, Kang SS, Yun CH, Oh JW, Seo HS and Han SH: Lipoteichoic acid of Enterococcus faecalis induces the expression of chemokines via TLR 2 and PAFR signaling pathways. J Leukoc Biol 94: 1275-1284, 2013.

30. Kuebler U, Zuccarella-Hackl C, Arpagaus A, Wolf JM, Farahmand F, von Känel R, Ehlert U and Wirtz PH: Stress-induced modulation of NF- $\kappa \mathrm{B}$ activation, inflammation-associated gene expression and cytokine levels in blood of healthy men. Brain Behav Immun 46: 87-95, 2015. 